\title{
Why does the sense of smell vanish in the mouth? Testing predictions from two accounts
}

\author{
Richard J. Stevenson • Mehmet Mahmut
}

Published online: 17 December 2014

(C) Psychonomic Society, Inc. 2014

\begin{abstract}
When participants perceive flavor they do not recognise the role of smell. We examined two possible accounts of why: (1) a common attentional channel activated by taste; and (2) prior learning between taste and smell. Participants were asked to sniff food-related odors with a fluid in their mouth and profile each odor after expectorating. This process was later repeated for each odor, with some odors experienced with water on both occasions, and others with water on one occasion and sucrose (weak or strong) on the other. We investigated how reliable these odor profiles were and whether they were influenced by prior odor-taste learning (indexed by odor sweetness). For non-sweet smells, the presence of a tastant significantly improved profile reliability relative to water in the mouth. For sweet smells, tastant had no effect, which we suggest represents a cancelling out of the beneficial effects of the common attentional channel by the detrimental effects of prior learning. Thus, both mechanisms may contribute to masking the modal identity of smell thereby contributing to flavor binding.
\end{abstract}

Keywords Taste $\cdot$ Smell $\cdot$ Flavor $\cdot$ Multisensory integration

\section{Introduction}

Multisensory integration may occur when stimuli share a common spatial location and time-course (e.g., Stein, Wallace \& Stanford, 2001). One example of this is flavor perception. Taste, olfactory, and somatosensory information arising from a common location in the mouth, and occurring over a similar time course, gives rise to flavor (e.g., Small \& Prescott, 2005).

R. J. Stevenson $(\bowtie) \cdot$ M. Mahmut

Department of Psychology, Macquarie University,

Sydney NSW2109, Australia

e-mail: dick.stevenson@mq.edu.au
The precise mechanics of flavor binding are a matter of debate, partly at least because some of its characteristics are unusual (Stevenson, 2014). In particular, olfaction has two modes of perception but only one set of receptors (Rozin, 1982). In one mode, odorants are perceived as smells arising in the environment when they are sniffed at the nose (orthonasal perception). In the other, odorants in food released by chewing stimulate the olfactory receptors via the internal nares (retronasal perception) and are described as "taste" or flavor.

What makes the distinction between these two modes of perception unusual is not the shift of perceived location (nose/mouth), of which there are other examples (e.g., ventriloquism; Bertelson \& Radeau, 1981), but the change in modality awareness. While the ventriloquist's voice may seem to arise from another source, it is still perceived as a sound. In contrast, for retronasal olfaction, although perceptual content is experienced (e.g., strawberryness), people do not recognise the modality from which it arises (Rozin, 1982).

As with other binding problems, there may be both peripheral and central causes. In this manuscript, we examined two centrally based accounts of flavor binding. One is that prior learning between particular tastes and smells in the mouth comes to blur their perceptual and modal boundaries (Prescott, 1999). For example, Dalton et al. (2000) found that a weak sweet taste, but not a non-sweet-taste, enhanced detection of a sweet-smelling odorant. As eating occurs in the mouth, and the mouth is the seat of taste (and flavor), the resulting percept may thus be called taste (or flavor). A further explanation is that gustatory stimulation in the mouth automatically activates a common attentional channel that encompasses both taste and smell (e.g., Auvray \& Spence, 2008; Gibson, 1966). In other words, smell with taste in the mouth is a gustatory percept.

If prior learning between taste and smell is important in masking modality awareness, then olfactory perception should be somewhat different for a smell perceived at the 
same time as its associated taste, relative to just smelling that odor alone. This is because the smell and taste will form a unitary percept that will differ from smelling that odor in the absence of that taste. For taste and smell pairs that have no such strong associative history, perception under these two conditions should not be so different.

Another prediction can be derived from the common attentional channel account. If water is present in the mouth, thus drawing attention to this locus, and a smell is present, perception of this smell should be poorer than when a taste is present in the mouth. This is because a taste may allow attention to smell during oral stimulation, resulting in enhanced perception relative to oral somatosensory stimulation alone (i.e., water). This contention is suggested by two pieces of evidence. First, fluids with little taste, such as water or viscous liquids in the mouth, act to reduce the perceived intensity of odors smelled at the nose or in the mouth, suggesting a reduced capacity to attend to the odor (e.g., Bult, de Wijk \& Hummel, 2007). Second, when a tastant is present, this can result in an increase in discriminability relative to water alone (e.g., Stevenson \& Mahmut, 2011).

To test these predictions, participants smelled an odor at the nose with a fluid in the mouth, profiling the odor (i.e., rating its qualities), and then later repeating this process to determine the reliability of the profile. Reliability has been used before to assess perceptual precision, both in vision (e.g., synaesthesia; Simner et al., 2005) and olfaction. For the latter, we have previously established that profile reliability is poorer when odors cannot be named consistently (Stevenson \& Mahmut, 2013a). In addition, profiles can later be matched at above chance levels to the odors from which the profiles were derived, and performance on this task is degraded the greater the interval between smelling the odor and making the profile (Stevenson \& Mahmut, 2013b). Together, these findings suggest that profile reliability can reflect how clearly the odor is perceived.

Smelling at the nose with fluids in the mouth was adopted to minimise the possibility of any physical interactions between tastant and odorant, a procedure used before in investigations of this type (e.g., Dalton et al., 2000; Sakai et al., 2001; Stevenson \& Mahmut, 2011). Crucially, this mode of presentation does not impair key phenomena associated with flavor binding (e.g., Dalton et al., 2000; Sakai et al., 2001). For example, the sweetness-enhancement effect (Frank \& Byram, 1988), whereby flavours composed of sweet-smelling odors and sweet tastes result in enhanced judgments of sweetness (Sakai et al., 2001) and facilitated detection of sweet smells when weak sweet tastes are present in the mouth (Dalton et al., 2000), all can occur with orthonasally presented odors.

The basic manipulation was to vary the fluid in the mouth. For some odors, the fluid would be water on one occasion and a tastant on another. For other odors, the fluid would always be water. The tastant used was sucrose, because this is a frequent associate of certain smells (Prescott, 1999). When asked to profile each odor, participants were asked to judge how similar the odor smelled to a set of seven olfactory descriptors (e.g., How banana-like does this smell?). The odorants selected were all food-related, and importantly we selected a number that we knew would probably have been experienced with a sweet taste. One method of gauging whether an odor has an association with a sweet taste is to determine how sweet smelling the odor alone is judged to smell. For this reason, sweetness ratings (alongside ratings to determine familiarity, intensity, liking, food-likeness and nameability) were obtained for all of the odors used in the experiment, so that we could select for each participant, smells that they perceived to be sweet or not-sweet smelling, thus reflecting their associative history with those smells.

We predicted that profile reliability would vary depending upon the odor and taste. First, for an odor smelled with sucrose in the mouth on one occasion and water on another (i.e., one less noisy profile vs. a moderately noisy profile), we predicted that the profiles would be more alike than for odors smelled with water in the mouth on both occasions (i.e., two moderately noisy profiles). In addition, we also manipulated tastant concentration, predicting that a stronger concentration should increase channel activation, thereby further enhancing reliability. Second, for odors that have an associative history with sucrose (sweet smells), this should result in a unified percept with a different profile to that of the odor alone in water, thus reliability should be poorer for such odors in sucrose, relative to water. Furthermore, it may be poorer still for the stronger concentration. Third, if both hypothesized mechanisms are at work, then we would expect smell type (i.e., sweet vs. notsweet) to moderate the effect of tastant, as the predicted detrimental effects of learning on reliability should offset the beneficial effects of a tastant for the sweet smells, but not for the not-sweet smells.

\section{Methods}

\section{Participants}

Thirty-nine people took part, 13 male, with a mean age of 23.4 $(\mathrm{SD}=7.9)$. A target of 40 participants was set based upon power calculations estimating a small to moderate effect size. All participants reported an intact sense of smell. Informed consent was provided by each participant and the protocol was approved by the university institutional review board.

Stimuli

Odorants were presented in opaque 200-ml, plastic, squeezable bottles. Table 1 details the concentrations and identities of the 18 odorants. Tastants were $4 \%$ (weak) and $16 \%$ (strong) 
Table 1 Odorants used in the experiment

\begin{tabular}{|c|c|}
\hline Name (quantity, source) & Name (quantity, source) \\
\hline Cupuacu (0.1 g; Dragoco) & Oil of cloves ( $0.07 \mathrm{~g}$; Sigma $)$ \\
\hline Guava (0.07 g; Dragoco) & Lemon oil (0.14 g; Sigma) \\
\hline Longan (0.13 g; Dragoco) & Vanilla (0.14 g; Dragoco) \\
\hline Water chestnut (0.07 g; Quest) & Mandarin (0.21 g; Quest) \\
\hline Lychee (0.07 g; Quest) & Grapefruit (0.17 g; Quest) \\
\hline Rose water (0.5 g; Essence) & Plum (0.07 g; Quest) \\
\hline Cherry (0.07 g; Quest) & Banana (0.1 g; Quest) \\
\hline Grape (0.15 g; Quest) & Pear (0.15 g; Quest) \\
\hline Kiwifruit (0.1 g; Dragoco) & Aniseed (0.15 g; Dragoco) \\
\hline
\end{tabular}

sucrose solutions (weight for volume). The profile rating sets used to evaluate each odorant were composed of seven scales. Each set of seven scales, used for a particular participant and odorant, were drawn randomly from a master set of 18 rating types (strawberry, banana, pineapple, peach, grape, orange, lychee, cherry, apple, lemon, melon, pear, kiwi, licorice, cloves, rose, vanilla, manderin). Participants were asked to judge, for each rating type, how $X$-like the odor smelled (e.g., How kiwi-like does this smell?), and rate this on a seven-point scale from not at all (0) to very (6).

\section{Procedure}

Participants completed the study in one session. The session was composed of two blocks of trials, separated by a 4-min break, with each block consisting of 18 trials. Each trial on one block had a counterpart on the other block, and each of these counterparts shared the same odor and the same profile rating set of seven scales. The 18 trials on a block consisted of 3 groups of 6 trial types, which are illustrated in Table 2. For trial types 3 and 4, and 5 and 6 (Table 2), order across blocks had no effect on reliability judgments. For each participant,

Table 2 Six trial types used in the experiment

\begin{tabular}{|c|c|}
\hline On the first block sniff... & $\begin{array}{l}\text { Then later on the second } \\
\text { block sniff, the same odor } \\
\text { with... }\end{array}$ \\
\hline $\begin{array}{l}\text { 1. Odor } 1 \text { with water \& judge } \\
\text { rating set A }\end{array}$ & Water \& judge rating set $\mathrm{A}$ \\
\hline $\begin{array}{l}\text { 2. Odor } 2 \text { with water } \& \text { judge } \\
\text { rating set B }\end{array}$ & Water \& judge rating set B \\
\hline $\begin{array}{l}\text { 3. Odor } 3 \text { with water } \& \text { judge } \\
\text { rating set } C\end{array}$ & $\begin{array}{l}\text { Weak sucrose \& judge } \\
\text { rating set } C\end{array}$ \\
\hline $\begin{array}{l}\text { 4. Odor } 4 \text { with weak sucrose } \\
\text { \& judge rating set } D\end{array}$ & Water $\&$ judge rating set $\mathrm{D}$ \\
\hline $\begin{array}{l}\text { 5. Odor } 5 \text { with water } \& \text { judge } \\
\text { rating set } \mathrm{E}\end{array}$ & $\begin{array}{l}\text { Strong sucrose \& judge } \\
\text { rating set } E\end{array}$ \\
\hline $\begin{array}{l}\text { 6. Odor } 6 \text { with strong sucrose } \\
\& \text { judge rating set } \mathrm{F}\end{array}$ & Water and judge rating set $\mathrm{F}$ \\
\hline
\end{tabular}

the 18 odorants were randomly assigned to these 18 trials, and 18 rating sets (each composed of seven scales) were randomly selected for each odor.

On each Block the trial structure was the same. Participants exhaled, poured a liquid into their mouth $(15 \mathrm{ml}$ of either water, weak or strong sucrose dependent on the trial type), and then sniffed the odor through their nose while the experimenter puffed it three times towards their nares. Once three large puffs had been presented, participants were asked to spit the liquid out, breathe normally, rinse their mouth with water, and then profile the odor on its particular set of seven ratings scales. A minimum of $30 \mathrm{sec}$ elapsed before the next trial commenced.

After both blocks were completed a final rating task took place. Each of the 18 odors was smelled in random order. For each one, an attempt was made at identification (guessing if no name was forthcoming) and then it was evaluated on five dimensions. In fixed order, participants rated: how much they liked or dislike the odor (dislike [0], indifference [3], like [6]), how familiar they were with it (never smelled before today [0], smelled a few times before [3], smelled many times before [6]), how strong it smelled (absent [0], slight [1], moderate [3], extreme [6]), how food-like it smelled (not at all [0] to very [6]) and how sweet it smelled (not at all [0] to very [6]).

\section{Analysis}

We started by calculating a reliability score between each trial with a particular odor on one block and its counterpart on the other block. This involved taking the mean absolute difference between the two sets of profile ratings for each odor, with larger values indicating greater disagreement. This score could range from 0 (i.e., perfect agreement) to 6 (i.e., perfect disagreement). In addition, we also calculated the number of nonzero scales for each trial with a particular odor on one block and its counterpart on the other block. This score could range from 0 (i.e., all 14 ratings across both trials received a rating of 0) to 14 (i.e., all 14 ratings across both trials received a rating of 1 or more). This non-zero score allowed us to check whether the same number of ratings was being used in the different conditions of the experiment.

Each participant had 18 reliability scores and 18 non-zero scores (i.e., one for each odor). We started by eliminating any odor trials where the participant had correctly named it, on the basis that these trials would likely be more consistent than those that were not named, thus contributing noise to the design. On average, each participant correctly named 3.4 odors ( $\mathrm{SD}=2.0$, range $0-9$ ). The named odors had a mean reliability score of $0.58(\mathrm{SD}=0.44)$ compared with all of the remaining trials where the odors could not be named, $\mathrm{M}$ $=0.86(\mathrm{SD}=0.45)$. The difference between these two means was significant, $\mathrm{t}(37)=5.48, p<0.001, \mathrm{~d}=$ 
0.89 , indicating that correctly named odors were more consistently profiled.

Once named odors were removed, reliability scores were organised by manipulation, namely water/water, weak sucrose/water, and strong sucrose/water. Each of these three sets of odors was then ranked by odor sweetness (participant-by-participant) and subject to median split to form a sweet and not-sweet set of odors for each manipulation. Mean reliability scores were then calculated, giving each participant six scores: (1) water/water and sweet smell; (2) water/water not-sweet smell; (3) weak sucrose/water sweet smell; (4) weak sucrose/water not-sweet smell; (5) strong sucrose/water sweet smell; and (6) strong sucrose/water not-sweet smell. In addition, and again for each participant, we calculated the non-zero scale score for each of these same six combinations. Next, we calculated the mean liking, familiarity, intensity, food-relatedness and sweetness scores for each of the six combinations above. All data from this study were analysed by repeated measures ANOVA, with trend test and planned comparisons as indicated by our hypotheses.

\section{Results}

We started by comparing the characteristics of the odors that composed each cell of the design (Table 3). The principal finding was that sweet smells were judged as smelling sweeter, more liked, familiar, intense and food-like, than notsweet smells.
Figure 1 presents the six mean reliability scores. A two-way repeated measures ANOVA of the reliability data with tastant (water/water vs. weak sucrose/water vs. strong sucrose/water) and odorant (smell sweet vs. not smell sweet), revealed a main effect of tastant, $\mathrm{F}(2$, $76)=3.87, p=0.025$, partial eta-squared $=0.09$, with a significant linear trend for this factor, $\mathrm{F}(1,38)=4.90, p$ $<0.05$, partial eta-squared $=0.11$. However, there also was a significant interaction of tastant by odorant, $\mathrm{F}(2$, $76)=3.22, p<0.05$, partial eta-squared $=0.08$, with a significant linear by linear trend for the interaction, $\mathrm{F}(1$, $38)=4.77, p<0.05$, partial eta-squared $=0.11$. This finding suggests that odor type moderates the effect of the tastant, implying that both processes are at work. Indeed, for not-sweet smells, reliability was poorer for water-water trials than for those smelled with a tastant, $\mathrm{t}(38)=2.71, p=0.01, \mathrm{~d}=0.43$, but this was not the case for sweet smells $(t=1.2)$.

Finally, we examined whether the non-zeros score varied by tastant and odorant using a further two-way repeated measures ANOVA. The ANOVA revealed no main effect of tastant $(F<1)$ and no interaction between tastant and odorant $(\mathrm{F}<1)$. However, there was a significant main effect of odorant, $\mathrm{F}(1,38)=8.32, p$ $<0.01$, partial eta-squared $=0.18$. As can be seen in Fig. 2, the sweet smelling odors had on average more non-zero descriptors than the non-sweet smelling odors. However, this difference was uniform across factor tastant, indicating that differences in the number of ratings cannot account for either the tastant or tastant by odorant effects observed in the reliability analysis.

Table 3 Means (and SDs) from the odor rating data, including ANOVA findings (tastant [water vs. weak sucrose vs. strong sucrose] by odorant [sweet odor vs. not-sweet odor])

Odor rating (and ANOVA findings)

\begin{tabular}{|c|c|c|c|c|c|}
\hline \multicolumn{2}{|l|}{ Water } & \multicolumn{2}{|c|}{ Weak sucrose } & \multicolumn{2}{|c|}{ Strong sucrose } \\
\hline Sweet odor & Not-sweet odor & Sweet odor & Not-sweet odor & Sweet odor & Not-sweet odor \\
\hline \multicolumn{6}{|c|}{ Sweetness (odorant, $\mathrm{F}(1,38)=532.62, p<0.001$, partial eta-squared $=0.90$ ) } \\
\hline $4.7(1.1)$ & $1.7(1.4)$ & $4.4(1.2)$ & $1.4(1.1)$ & $4.5(1.0)$ & $1.5(1.1)$ \\
\hline \multicolumn{6}{|c|}{ Liking (odorant, $\mathrm{F}(1,38)=102.19, p<0.001$, partial eta-squared $=0.62$ ) } \\
\hline $4.1(1.2)$ & $2.7(1.0)$ & $4.2(1.1)$ & $2.6(1.3)$ & $4.1(1.2)$ & $3.0(1.2)$ \\
\hline \multicolumn{6}{|c|}{ Familiarity (odorant, $\mathrm{F}(1,38)=27.56, p<0.001$, partial eta-squared $=0.42 ;$ and odorant and tastant, $\mathrm{F}(2,76)=3.60, p<0.05$, partial eta-squared $=0.09^{1}$ ) } \\
\hline $3.9(1.2)$ & $2.6(1.4)$ & $3.7(1.2)$ & $2.7(1.4)$ & $3.6(1.1)$ & $3.2(1.2)$ \\
\hline \multicolumn{6}{|c|}{ Intensity (odorant, $\mathrm{F}(1,38)=12.83, p<0.001$, partial eta-squared $=0.25$ ) } \\
\hline $4.1(0.9)$ & $3.4(1.0)$ & $4.0(1.0)$ & $3.7(0.9)$ & $4.2(0.8)$ & $3.8(0.8)$ \\
\hline \multicolumn{6}{|c|}{ Food-likeness (odorant, $\mathrm{F}(1,38)=97.89, p<0.001$, partial eta-squared $=0.41$ ) } \\
\hline $3.7(1.7)$ & $2.2(1.5)$ & $3.5(1.5)$ & $2.2(1.6)$ & $3.5(1.4)$ & $2.4(1.7)$ \\
\hline
\end{tabular}

${ }^{1}$ Bonferroni corrected post-hoc contrasts revealed no significant differences 


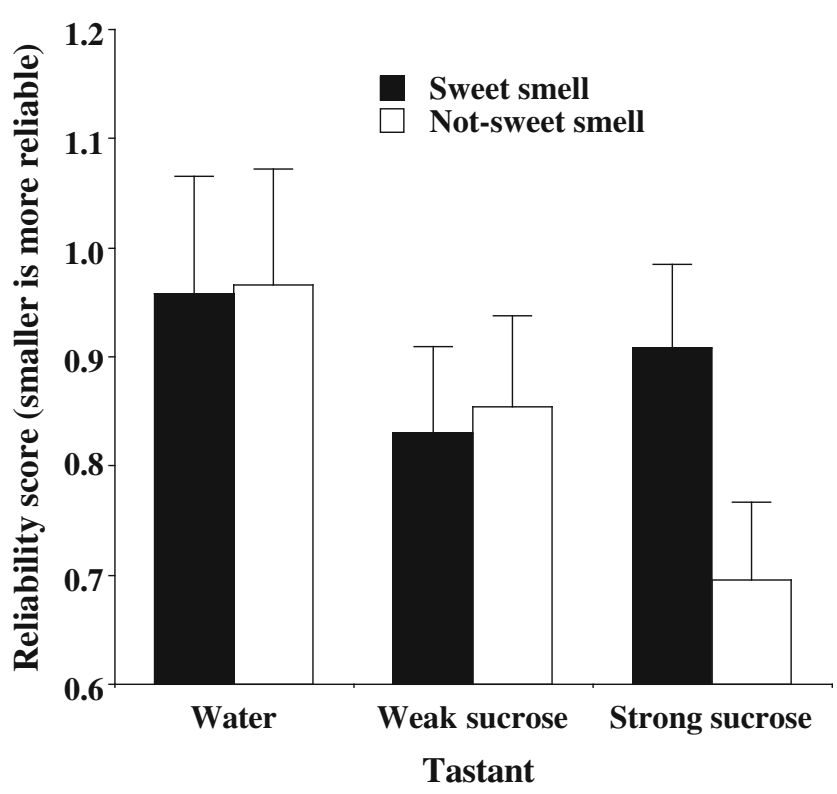

Fig. 1 Mean (and standard error) reliability scores, with lower score indicating greater reliability, for sweet and not-sweet odors smelled while different fluids (tastants) were present in the mouth

\section{Discussion}

Taste, olfaction, and somatosensation combine to form flavor. One consequence of this is that most people do not seem aware that olfaction is involved in their flavor experience (Rozin, 1982). We tested two centrally based accounts of flavor binding. One predicted that the presence of a taste in the mouth should automatically activate a shared gustatoryolfactory channel, resulting in improved odor perception. The other predicted that odors with history of co-occurrence with

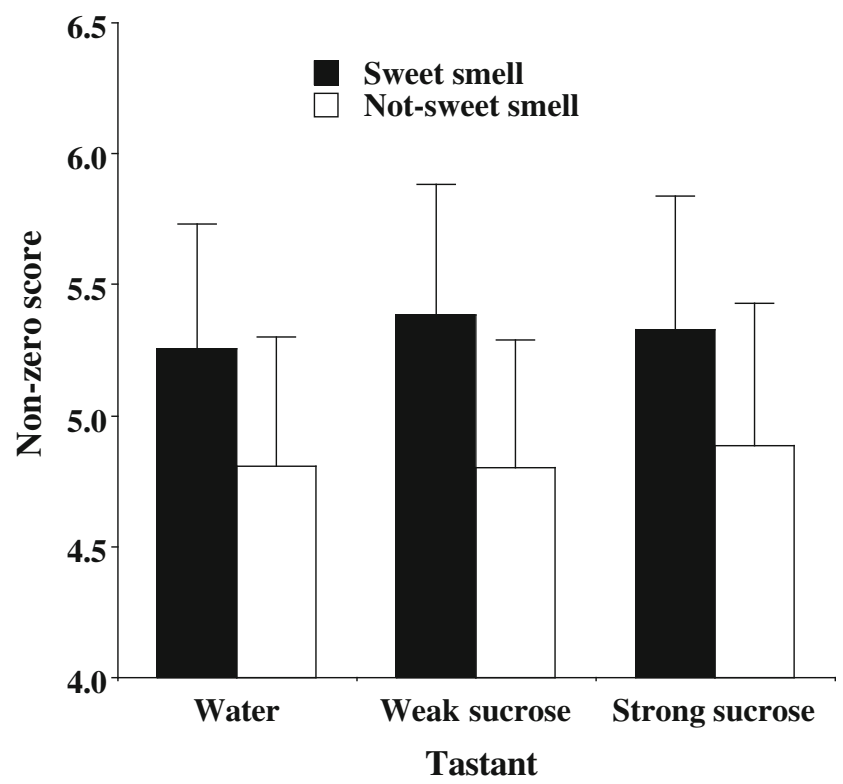

Fig. 2 Mean (and standard error) non-zero rating scores, indicating the number of ratings given a response of one or more, for sweet and not-sweet odors smelled while different fluids (tastants) were present in the mouth sweet tastes should, when combined with a sweet taste be perceived as an object different to that of the smell alone. We further predicted that if both of these mechanisms were present, then the type of odor (sweet vs. not-sweet) should moderate the effect of taste on odor profile reliability. In this case, the detrimental effects of taste-smell fusion for sweet odors should offset the advantages of a tastant activated attentional channel, but not for the not-sweet smells. Our findings were most consistent with both mechanisms working. For sweet smells, tastant had no discernible effect, which we suggest may represent the cancelling out of the beneficial effects of the sensory channel versus the detrimental effects of the discrepancy produced between the sweet odor-taste combination and the odor alone. For not-sweet smells, the presence of a tastant improved profile reliability.

The experiment described here used a novel combination of techniques and so it may be that its findings reflect the peculiarities of its procedures rather than revealing more general facts about lack of modality awareness and the interaction of taste and smell. Four issues seem important. The first is interpretive and concerns what profile reliability actually means in relation to odor perception. Odor profiles have frequently been used as a descriptive tool to capture an odor's quality (e.g., Dravnieks, 1982). These descriptions are stable and relate to participants ability to detect differences between pairs of odors (e.g., Royet et al., 1996). This implies that odor profiles capture aspects of odor quality, difference, and hence discriminability. That differences in profile reliability probably reflect differences in discriminability is further suggested by the finding here that greater reliability was associated with nameable odors. Not only does this mirror prior findings (Stevenson \& Mahmut, 2013a), it also directly parallels results from odor discrimination studies, which demonstrate enhanced discriminative ability with named stimuli (e.g., Rabin, 1988). In the present context, it suggests that where profile reliability is better, participants are more clearly able to perceive the odor when the tastant is present.

A second issue concerns generalising from orthonasal odorants to the natural flavor situation where both the odor and taste are in the mouth. We note that odor localisation to the mouth can still be obtained when an odor is presented at the nose with a taste in the mouth and that many phenomena observed with odors in the mouth can be obtained by presenting them at the nose with tastes in the mouth (e.g., Dalton et al., 2000; Sakai et al., 2001; Stevenson \& Mahmut, 2011; Stevenson, Oaten \& Mahmut, 2011). On this basis, we would suggest that our findings should still provide insight into how lack of olfactory modality awareness arises during flavor perception.

A third issue concerns the number of non-zero ratings made for each odor. The more ratings that are above zero, the more likely a different profile may emerge upon subsequent exposure to the same odor. If trials with a taste involved 
fewer or greater numbers of non-zero responses relative to trials with water, this could potentially account for differences in reliability observed for factor tastant. However, we found no net change in the number of non-zero ratings across the three levels of factor tastant, suggesting that the presence of a taste, relative to water, did not increase or reduce the number of non-zero ratings that participants provided. Similarly, there was no variation in the number of non-zero ratings by factor odorant at each level of factor tastant, although sweet odors in general were judged to have more non-zero qualities than nonsweet odors. Importantly then, the number of non-zero ratings remained stable for the two significant reliability effects (i.e., tastant, and tastant by odorant).

The fourth issue concerns other differences that were identified between the sweet and not-sweet odors. As the rating analysis revealed, sweet odors also were judged to smell more intense, more familiar, more pleasant and more food-like than not-sweet smells. These differences are not unique to this study and reflect characteristics that have been noted before to covary with odor sweetness (e.g., Stevenson, Boakes \& Wilson, 2000). However, what is particularly at issue here is whether the tastant by odorant interaction effect observed in the reliability data can be better explained with reference to odor sweetness than it can by reference to any of the other characteristics that covaried with this attribute. In this regard, a possible alternative candidate would be odor liking, which has been suggested before as a means of explaining perceptual interactions between tastes and smells (e.g., Prescott, 1999).

Determining how retronasal odor perception results in an apparent lack of modality awareness has not attracted much research attention (Stevenson, 2014). It is an interesting problem because as noted in the Introduction, its manifestation as loss of modality awareness contrasts with what is observed for other examples of cross-modal binding. While this may be the case, the mechanisms our findings favour broadly parallel explanations advanced for other forms of cross-modal binding, with their focus on centrally based attentional processes (e.g., Stein, Wallace \& Stanford, 2001) and more recently on the role of learning (e.g., Vatakis \& Spence, 2007).

The findings here also inform us about the nature of flavor binding. We have inferred from our results that sweet tastes probably enhance attention to the not-sweet smells. This would suggest that the putative joint (smell-taste) attentional channel initiated by taste in the mouth may be hardwired. In other words, it can operate irrespective or not of whether the taste and the odor have occurred together before, this being an equally plausible alternative (Stevenson, 2014). In conclusion, this would suggest that odor-taste experience and a possibly hardwired joint attentional channel might both contribute to participants oft cited failure to notice smell in the mouth.

\section{References}

Auvray, M., \& Spence, C. (2008). The multisensory perception of flavor. Consciousness and Cognition, 17, 1016-1031.

Bertelson, P., \& Radeau, M. (1981). Cross-modal bias and perceptual fusion with auditory-visual spatial discordance. Perception and Psychophysics, 29, 578-584.

Bult, J. H. F., de Wijk, R. A., \& Hummel, T. (2007). Investigations on multimodal sensory integration: Texture, taste, and ortho- and retronasal olfactory stimuli in concert. Neuroscience Letters, 411, 6-10.

Dalton, P., Doolittle, N., Nagata, H., \& Breslin, P. (2000). The merging of the senses: Integration of subthreshold taste and smell. Nature Neuroscience, 3, 431-432.

Dravnieks, A. (1982). Odor quality: Semantically generated multidimensional profiles are stable. Science, 218, 799-801.

Frank, R., \& Byram, J. (1988). Taste-smell interactions are tastant and odorant dependent. Chemical Senses, 13, 445-455.

Gibson, J. J. (1966). The senses considered as perceptual systems. London: Allen \& Unwin.

Prescott, J. (1999). Flavour as a psychological construct: Implications for perceiving and measuring the sensory qualities of foods. Food Quality and Preference, 10, 349-356.

Rabin, M. D. (1988). Experience facilitates olfactory quality discrimination. Perception and Psychophysics, 44, 532-540.

Royet, J., Paugam-Moisy, H., Rouby, C., Zighed, D., Nicoloyannis, N., Amghar, S., \& Sicard, G. (1996). Is short term odour recognition predictable from odour profile? Chemcial Senses, 21, 553-566.

Rozin, P. (1982). "Taste-smell confusions" and the duality of the olfactory sense. Perception and Psychophysics, 31, 397-401.

Sakai, N., Kobayakawa, T., Gotow, N., Saito, S., \& Imada, S. (2001). Enhancement of sweetness ratings of aspartame by a vanilla odor presented either by orthonasal or retronasal routes. Perception and Motor Skills, 92, 1002-1008.

Simner, J., Ward, J., Lanz, M., Jansari, A., Noonan, K., Glover, L. \& Oakley, D. A. (2005). Non-random associations of graphemes to colours in synaesthetic and non-synaesthetic populations. Cognitive Neuropsychology, 22, 1069-1085.

Small, D., \& Prescott, J. (2005). Odor/taste integration and the perception of flavor. Experimental Brain Research, 166, 344-357.

Stein, B. E., Wallace, M. T., \& Stanford, T. R. (2001). Brain mechanisms for synthesizing information from different sensory modalities. In B. Goldstein (Ed.), Blackwell handbook of perception (pp. 709-736). MA: Blackwell.

Stevenson, R. J. (2014). Flavor binding: Its nature and cause. Psychological Bulletin, 140, 487-510.

Stevenson, R. J., \& Mahmut, M. (2011). Olfactory test performance and its relationship with the perceived location of odors. Attention, Perception and Psychophysics, 73, 1966-1973.

Stevenson, R. J., \& Mahmut, M. (2013a). Using response consistency to probe olfactory knowledge. Chemical Senses, 38, 237-249.

Stevenson, R. J., \& Mahmut, M. (2013b). Familiarity influences odor memory stability. Psychonomic Bulletin and Review, 20, 754-759.

Stevenson, R. J., Boakes, R. A. \& Wilson, J. P. (2000). The persistence of conditioned odor perceptions: Evaluative conditioning is not unique. Journal of Experimental Psychology: Learning, Memory and Cognition, 26, 423-440.

Stevenson, R. J., Oaten, M., \& Mahmut, M. (2011). The role of taste and oral somatosensation in olfactory localisation. Quarterly Journal of Experimental Psychology, 64, 224-240.

Vatakis, A., \& Spence, C. (2007). Crossmodal binding: Evaluating the "unity assumption" using audiovisual speech stimuli. Perception and Psychophysics, 69, 744-756. 